Bitlis Eren Univ J Sci \&

Technol

6(2), 76-84, 2016

E - ISSN 2146-7706

\author{
Research Article
}

\title{
The Modeling and Performance Comparison of PD and Takagi-Sugeno Type Fuzzy Logic Controllers for Inverted Pendulum System
}

\author{
Sami Şit 1, Erdal Kılıç³, Hasan Rıza Özçalık², Hussein Alruim Alhasan², Ahmet Gani²,* \\ ${ }^{1}$ Department of Electrical and Electronic Engineering, Hakkari University, Hakkari, Turkey \\ ${ }^{2}$ Department of Electrical and Electronics Engineering, Kahramanmaraş Sutcu Imam University, Kahramanmaraș, \\ Turkey. \\ ${ }^{3}$ Department of Electrical and Energy, Afsin Vocational School, Kahramanmaras Sutcu Imam University, \\ Kahramanmaraş, Turkey. \\ ${ }^{*}$ Corresponding Author:ahmetgani8733@gmail.com
}

\begin{abstract}
In recent years, fuzzy logic based modeling and control methods have been increasingly used in the academic field. Inverted pendulum system consists of conventional materials with nonlinear dynamic structure in order to test types of controllers. This paper presents a simulation study of Takagi-Sugeno type fuzzy logic controllers for an inverted pendulum (cart-pole) system. A Takagi-Sugeno method was applied to the inverted-pendulum system, which can balance the pendulum over a greater range of pole angle and cart position. Takagi-Sugeno fuzzy logic controller proposed in this study is compared with the conventional PD controller in order to demonstrate the performance of the proposed controller. This study aims to determine the best control strategy that provides better performance in terms of pendulum's angle and cart's position. A Takagi-Sugeno type fuzzy logic controller yields better results. Simulation results demonstrated that the proposed fuzzy controller is effectiveness and robust.
\end{abstract}

Keywords: Takagi-Sugeno Fuzzy Logic Controller, PD Controller, Inverted Pendulum 


\section{E - ISSN 2146-7706}

\section{Introduction}

A Cart-Pole or Cart-Inverted pendulum system is one of the common problems of nonlinear control. It is extensively used as a benchmark to test different control algorithms (Yamakawa, 1989; Khosla, 2014). An unstable system, inverted pendulum system is one of the most significant problems in the field of control engineering. The dynamics which balance a pendulum at an unstable position are used to control various applications such as walking robots, helicopters, satellites, and space crafts.

Fuzzy logic offers a solution to this problem as humans can use their experience in order to design the controller. In general, fuzzy controllers consist of three stages: an input stage, a processing stage, and an output stage. The input stage maps inputs to the membership functions between 0 and 1 . The processing stage generates a result for each rule in accordance with inputs. Afterwards, it collects the results for each rule. Finally, the output stage converts the collected results to a truth control output value.

The task of the controller in the closed loop systems is to compare the output signal sent by the feedback with the input signal and thus create a suitable control signal depending on the error signal and controlling structure. There are three fundamental control effects used for conventional control systems: proportion (P) effect, integral (I) effect and derivative (D) effect. PD controller is used to minimize the system setting time, overshoot and instability (Bhattacharya, 2003).

The objective of the inverted pendulum system is to keep the pendulum in a vertically upward state or balanced state and to track the cart to a desired position despite the external disturbance. A feedback control system must be used to stabilize the inverted pendulum system and maintain the balanced state. The two control approaches that will overcome this problem are Takagi-Sugeno Type Fuzzy Logic (FLC) control and Proportional-Derivative (PD) control, which require being familiar with the system dynamics to obtain the best performance.

This paper presents a mathematical modeling and analysis of performance comparison between conventional PD controller and Takagi-Sugeno type fuzzy logic controller for an inverted pendulum system (Gani et al., 2014a; Gani et al., 2016).

\section{Mathematical Model of Inverted Pendulum}

The inverted pendulum (a cart and pole) system consists of a pendulum whose center of mass is above its pivot point, which is mounted on the motor-driven cart. The cart can move horizontally by a force $u$. The schematic diagram of the inverted pendulum or a cart and pole system is shown in Fig. 1 where $M$ is the mass of the cart, $m$ is the mass of the pendulum, $g$ is the acceleration of gravity, $l$ is the distance between the pivot and the mass center of the ball, $u$ is the force applied to the cart, $\theta$ is the pendulum angle from vertical, and $x$ is the cart position coordinate (Gani et al., 2016). The physical parameters of the inverted pendulum system are presented in Table 1 .

The inverted pendulum system is one of the most difficult non-linear systems. This system is unstable without control, and the pendulum will go down if the cart is not moved to balance it. A motor (in the cart) moves on the cart with a force $u$.. This force is manipulated by the controller to stabilize the pendulum in an upright state at a specific position of the cart (Gani et al., 2014b).

The inverted pendulum system has one input and four outputs. As input, the applied force $(u)$ enables the cart to move horizontally. The position of the cart, the velocity of the cart, the angle of the pendulum with the vertical line, and angular velocity of the pendulum are the system's outputs.

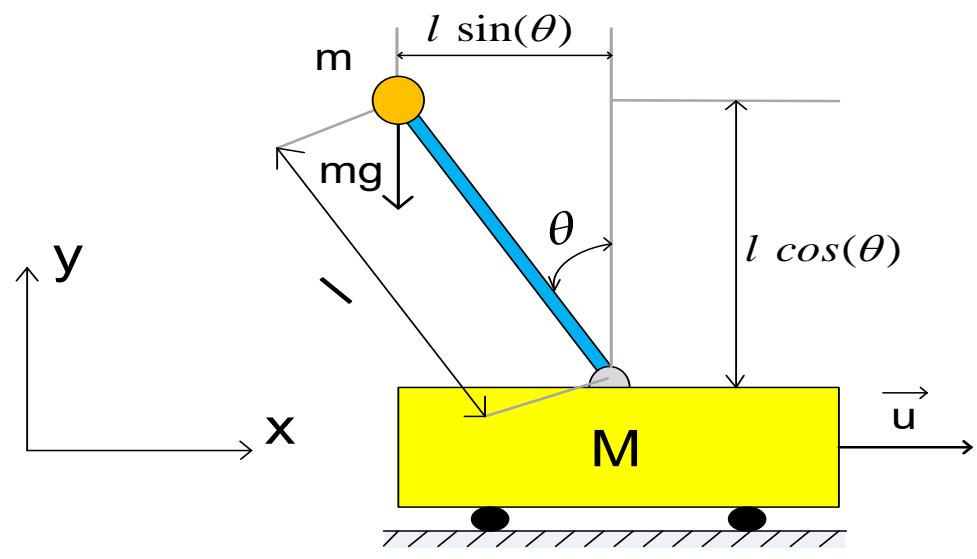

Fig 1.The inverted pendulum (a cart and pole) system.

The dynamics of the inverted pendulum system are non-linear. A mathematical model of the non-linear system is described below in accordance with the potential and kinetic energy of the system. In general,

Received June 19, 2016 Available online December, 2016 
Sit at al. / Bitlis Eren Univ J Sci \& Technol / 6(2), 76-84, 2016

the mathematical model of mechanical systems is usually defined by the Newtonian or Euler-Lagrange equations (Goher et al., 2010). Therefore, we can obtain the system dynamic equations through EulerLagrange method by calculating the difference between potential and kinetic energy. Thus, the final equations of motion for the inverted pendulum on a moving cart can be defined as:

$$
\begin{aligned}
& (M+m) \ddot{x}-m l \sin (\theta) \dot{\theta}^{2}+m l \cos (\theta) \ddot{\theta}=u \\
& m \ddot{x} \cos (\theta)+m l \ddot{\theta}=m g \sin (\theta)
\end{aligned}
$$

The equations (1) and (2) above show the dynamics of the nonlinear inverted pendulum system. To obtain the nonlinear state-space model of the system, we will define state vector as follows:

$$
\mathrm{x}=\left[\begin{array}{c}
\mathrm{x}_{1} \\
\mathrm{x}_{2} \\
\mathrm{x}_{3} \\
\mathrm{x}_{4}
\end{array}\right]=\left[\begin{array}{c}
\theta \\
\dot{\theta} \\
\mathrm{x} \\
\dot{\mathrm{x}}
\end{array}\right]
$$

Therefore,

$$
\begin{aligned}
& \dot{\mathrm{x}}_{1}=\mathrm{X}_{2} \\
& \dot{x}_{2}=\frac{-m l \sin \left(x_{1}\right) \cos \left(x_{1}\right) x_{2}^{2}+(M+m) g \sin \left(x_{1}\right)-u \cos \left(x_{1}\right)}{l\left[M+m-m \cos ^{2}\left(x_{1}\right)\right]} \\
& \dot{\mathrm{X}}_{3}=\mathrm{X}_{4} \\
& \dot{x}_{4}=\frac{m l \sin \left(x_{1}\right) x_{2}^{2}-m g \sin \left(x_{1}\right) \cos \left(x_{1}\right)+u}{M+m-m \cos ^{2}\left(x_{1}\right)}
\end{aligned}
$$

These equations describe the nonlinear state-space model of the inverted pendulum system.
Table 1. Physical Parameters of the Inverted Pendulum System.

\begin{tabular}{|c|c|c|c|}
\hline Parameter & Symbol & Value & Unit \\
\hline Mass of cart & $\mathrm{M}$ & 3 & $\mathrm{~kg}$ \\
\hline Mass of pendulum & $\mathrm{m}$ & 0.5 & $\mathrm{~kg}$ \\
\hline Length of pendulum & $\mathrm{l}$ & 0.5 & $\mathrm{~m}$ \\
\hline Acceleration of gravity & $\mathrm{g}$ & 9.8 & $\mathrm{~m} / \mathrm{s}^{2}$ \\
\hline
\end{tabular}

\section{PD Controller Design}

The PD control is a common strategy in controlling an inverted pendulum system (Kilic, 2015; Hasan, 2012). PD controller is a type of feedback controllers with a single output, which is a control variable $(\mathrm{u})$, and a single input, which is the error (e) between a reference variable $(r)$ and measured process variable (y). The block diagram of the PD controller that balances an inverted pendulum system is shown in Fig. 2. The general form of the PD algorithm is:

$$
u=k_{i} e(t)+k_{d} \dot{e}(t)
$$

Here, $K_{p}$ and $K_{d}$ denote the proportional and derivative gains, which are the tuning parameters, respectively.

In this study, one PD controller is required to control the position of the cart $(x)$ and another one to control the angle of the pendulum $(\theta)$. The block diagram of the PD controller for the inverted pendulum system is shown in Fig. 2. The PD controller calculates the error value $(e)$, which is the difference between a reference variable and measured process variable, and minimizes this error over time by modifying the control variable $(u)$. The error value for the first PD is equal to the current angle of the pendulum because the reference angle of the pendulum is set to ' 0 ' degree. However, the error value for the second PD is the difference between the desired position of the cart and current position. The control variable $(\mathrm{u})$ is the sum of the control variables $\left(u_{1}\right)$ and $\left(u_{2}\right)$ for both PD controllers (Gani et al., 2014a; Gani et al., 2016).

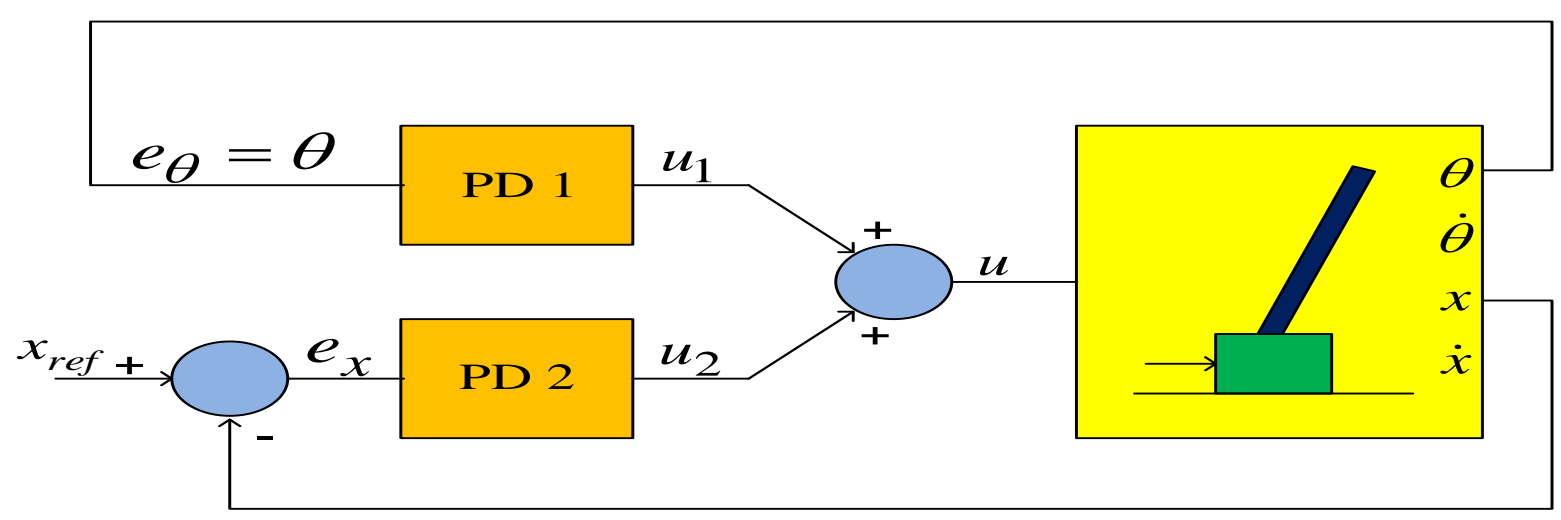

Figure 2. The block diagram of the PD controller. 


\section{E - ISSN 2146-7706}

Because derivative control reduces oscillation during transient regime and decreases the overshoot value caused by proportional control, the simultaneous use of proportional control and derivative control create efficient controlling structure non-linear dynamic systems such as an inverted pendulum. It is proposed that the derivative proportional controller will reduce overshoot value and increase the response speed of the system. This is why a PD controller was preferred. The gain parameters of PD controllers, which were designed to control the angle of inverted pendulum, and the position of the cart was calculated based on the reaction speed, settling time and stability of the controllers. The tuning process for the two proportional and derivative gains demonstrates that the best PD controller responses for position of the cart and angle of the pendulum are achieved by setting $K_{p 1}=70, K_{d 1}=20$ for the first PD controller and $K_{p 2}=4$, and $K_{d 2}=6$ for the second PD controller. These parameters must be simultaneously tuned in order to reach the perfect system response.

\section{Takagi-Sugeno Type Fuzzy Logic Controller Design}

Takagi-Sugeno fuzzy model is a well-known method of fuzzy reasoning and used in many applications in fuzzy control and fuzzy systems (Takagi, 1985; Wang, 2015). The Takagi-Sugeno fuzzy controller possesses output, which is the control variable or the applied force $(u)$, and four inputs, which are the error in the pendulum angle $e(\theta)$, the angular velocity of the pendulum, the error between a desired position and the current position of the cart $e(x)$, and the velocity of the cart. These four input variables heavily influence the control variable $(u)$ applied to the cart. The accuracy of membership functions (MFs) and rule base of the T-S fuzzy controller remarkably improves the performance of the system (Sugeno, 1985; Marzi, 2005; Acikgoz, 2014).

Five linguistic variables are used in this study for each controller input: NB (negative big), NS (negative small), ZE (zero), PS (Positive small), and PB (positive big). NB and PB display a sigmoid membership function while NS, ZE, and PS display a Gaussian membership function (Lee, 1990; Ozcalik, 2014).

The sigmoid membership function is defined by the following equation:

$$
\operatorname{sigmoid}(x ; a, c)=\frac{1}{1+e^{-a(x-c)}}
$$

It depends on $(a)$ and $(c)$ where $(c)$ represents the crossover point of the sigmoid membership function and $(a)$ determines whether the sigmoid membership function is open left or right.

On the other hand, the symmetric Gaussian membership function also depends on two parameters $(\sigma, \mathrm{c})$ and is in the following form:

$$
\operatorname{gaussian}(\mathrm{x} ; \sigma, \mathrm{c})=\mathrm{e}^{\frac{-(\mathrm{x}-\mathrm{c})^{2}}{2 \sigma^{2}}}
$$

Here, (c) represents the center of the Gaussian membership function and $(\sigma)$ determines the width of the Gaussian membership function (Years, 2014).

Figs. 3-6 shows the membership function used for each input of the inverted pendulum system.

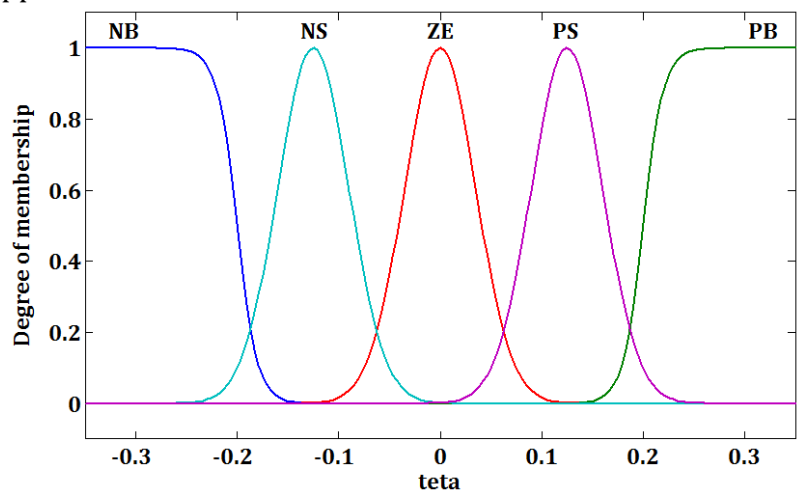

Figure 3. Membership functions for the error in the angle of the pendulum. 
Sit at al. / Bitlis Eren Univ J Sci \& Technol / 6(2), 76-84, 2016

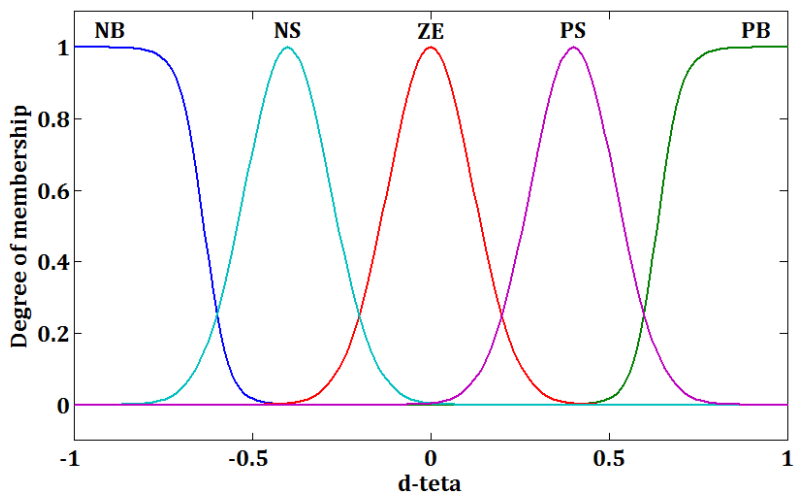

Figure 4. Membership functions for the angular velocity of the pendulum.

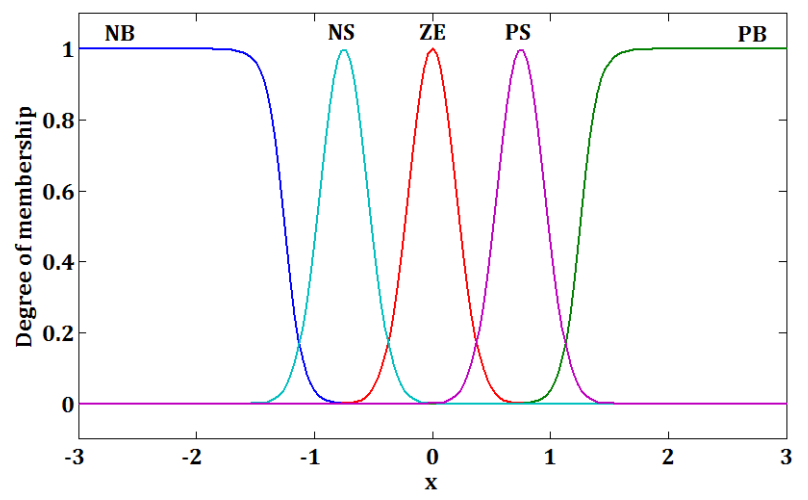

Figure 5. Membership functions for the error in the position of the cart.

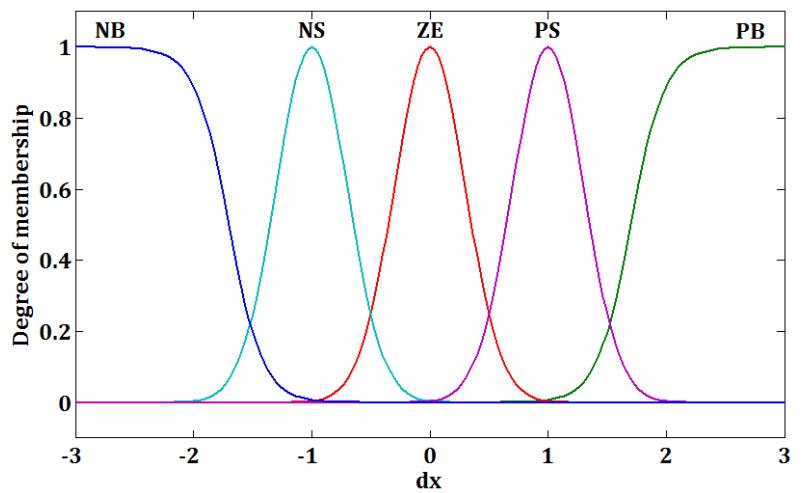

Figure 6. Membership functions for the velocity of the cart.

In general, the fuzzy control is a rule-based control system. Fuzzy rules play an important role in linking the input variables of the fuzzy controller, the inverted pendulum system, to the output variable, the control signal $(\mathrm{u})$. We defined IF-THEN rules to control the position of the cart and angle of the pendulum. The total number of IF-THEN rules is 50 .
There are 25 rules to keep the pendulum in upward state and 25 rules to track the cart to a desired position (Sevil, 2015).

A complete set of IF-THEN rules that controls the whole system is presented in Table 2 and Table 3, respectively.

Table 2. Complete set of rules for the position of the cart

\begin{tabular}{|c|c|c|c|c|c|c|}
\hline \multicolumn{2}{|c|}{} & \multicolumn{5}{|c|}{$\dot{x}$} \\
\cline { 3 - 7 }$z$ & NB & NS & ZE & PS & PB \\
\hline & NB & SB & SB & SM & SM & SS \\
\hline
\end{tabular}


Sit at al. / Bitlis Eren Univ J Sci \& Technol / 6(2), p-p, 2016

\begin{tabular}{|c|c|c|c|c|c|c|}
\hline \multirow{3}{*}{$e_{x}$} & NS & SB & SM & SM & SS & SS \\
\cline { 2 - 7 } & ZE & SM & SS & SS & SS & SM \\
\cline { 2 - 7 } & PS & SS & SS & SM & SM & SB \\
\cline { 2 - 7 } & PB & SS & SM & SM & SB & SB \\
\hline
\end{tabular}

Table 3. Complete set of rules for the angle of the pendulum.

\begin{tabular}{|c|c|c|c|c|c|c|}
\hline \multicolumn{2}{|c|}{} & \multicolumn{5}{|c|}{$\dot{\theta}$} \\
\cline { 2 - 7 } \multicolumn{2}{|c|}{} & NB & NS & ZE & PS & PB \\
\hline \multirow{4}{*}{} & NB & BS & BS & MS & MS & SS \\
\cline { 2 - 7 }$e_{\theta}$ & NS & BS & MS & MS & SS & SS \\
\cline { 2 - 7 } & ZE & MS & SS & SS & SS & MS \\
\cline { 2 - 7 } & PS & SS & SS & MS & MS & BS \\
\cline { 2 - 7 } & PB & SS & MS & MS & BS & BS \\
\hline
\end{tabular}

The output level (z) displays five membership functions: SB (small big), SM (small medium), SS (small small), MS (medium small), and BS (big small). Each membership function refers to linear equation for the output level (z).

For example, the rule number ' 1 ' for the position of the cart is in the following form:

$$
\begin{aligned}
\text { IF } e_{x}= & N B \text { and } \dot{x}=N B \text { Then } \\
& z_{1}=S B=a_{1} \theta+b_{1} \theta+c_{1} x+d_{1} x+r_{1}
\end{aligned}
$$

The output level z1 must be (SB), i.e. small big, for the angle of the pendulum coefficients and big for the position of the cart coefficients. If both error of the position and velocity of the cart are (NB) or negative big, the cart is located in the left and the cart is going to the left.

The parameter vector [ ] for each membership function is tuned by trial-and-error method to achieve an acceptable performance. The best parameter vector values for each membership function are

\begin{tabular}{|c|c|c|c|c|c|}
\hline SB & $=[155$ & 55 & 15 & 40 & 0] \\
\hline BS & $=[200$ & 70 & 5 & 15 & 0] \\
\hline SS & $=[155$ & 55 & 5 & 15 & 0] \\
\hline SM & $=[155$ & 55 & 10 & 20 & 0] \\
\hline MS & $=[180$ & 60 & 5 & 15 & 0] \\
\hline
\end{tabular}
presented as follows:

In the following step, the output level of each rule is weighted by the firing strength of the rule. The firing strength for an AND rule with 4 inputs [ $\left.\begin{array}{llll}\theta & \dot{\theta} & x & \dot{x}\end{array}\right]$ is given by:

$\mathrm{w}_{\mathrm{i}}=\operatorname{AndMethod}\left(\mu_{\mathrm{i}}(\theta), \mu_{\mathrm{i}}(\dot{\theta}), \mu_{\mathrm{i}}(\mathrm{x}), \mu_{\mathrm{i}}(\dot{\mathrm{x}})\right)$

Here $\left[\mu_{i}(\theta), \mu_{i}(\dot{\theta}), \mu_{i}(\mathrm{x}), \mu_{i}(\dot{\mathrm{x}})\right]$ are the degrees of membership functions for system inputs [ $\left.\begin{array}{llll}\theta & \dot{\theta} & x & \dot{x}\end{array}\right]$, respectively.

The control signal $(\mathrm{u})$ or the final output of the proposed controller is the weighted average of all rule outputs obtained by using center of area defuzzification strategy, and is defined by the following equation:

$$
u=\frac{\sum_{i=1}^{N} w_{i} z_{i}}{\sum_{i=1}^{N} w_{i}}
$$

where $(\mathrm{N}=50)$ represents the number of the used rules (Hellendom, 1993).

\section{Simulation Results}

In this section, a comparison of the conventional PD and a Takagi-Sugeno type fuzzy logic controller based on simulation results in the presence and absence of disturbance are discussed. Both PD controller and a Takagi-Sugeno type fuzzy controller for the inverted pendulum system are modeled and simulated in the MATLAB/Simulink. 
The time response of pendulum angle and cart position with both PD and fuzzy controllers without disturbance for an initial pendulum angle of $10^{\circ}$ and a cart reference position of 1 meter are shown in Fig.8 and Fig. 9, respectively. The influence of disturbance at the time response of pendulum angle and cart position is shown in Fig. 10 and Fig. 11, respectively.

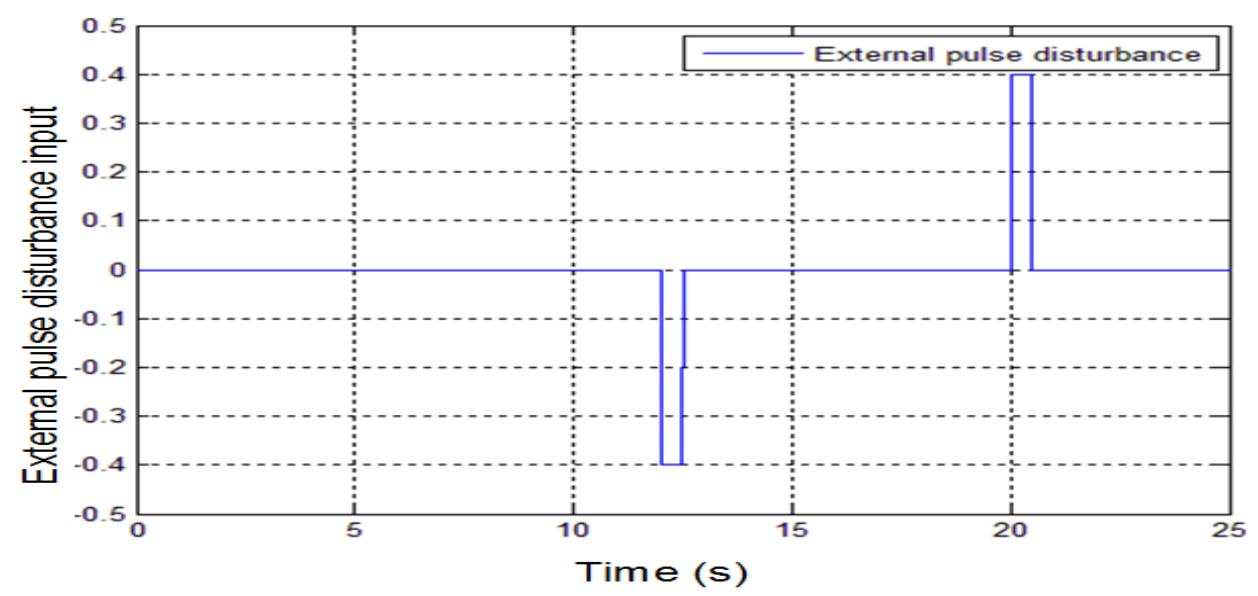

Figure 7. External pulse disturbance input.

$0.4 \mathrm{~N}$ external pulse disturbance input was applied to the system in 12. sec. and 20. sec. in the opposite direction. External pulse disturbance input designed for system is shown in Fig. 7.

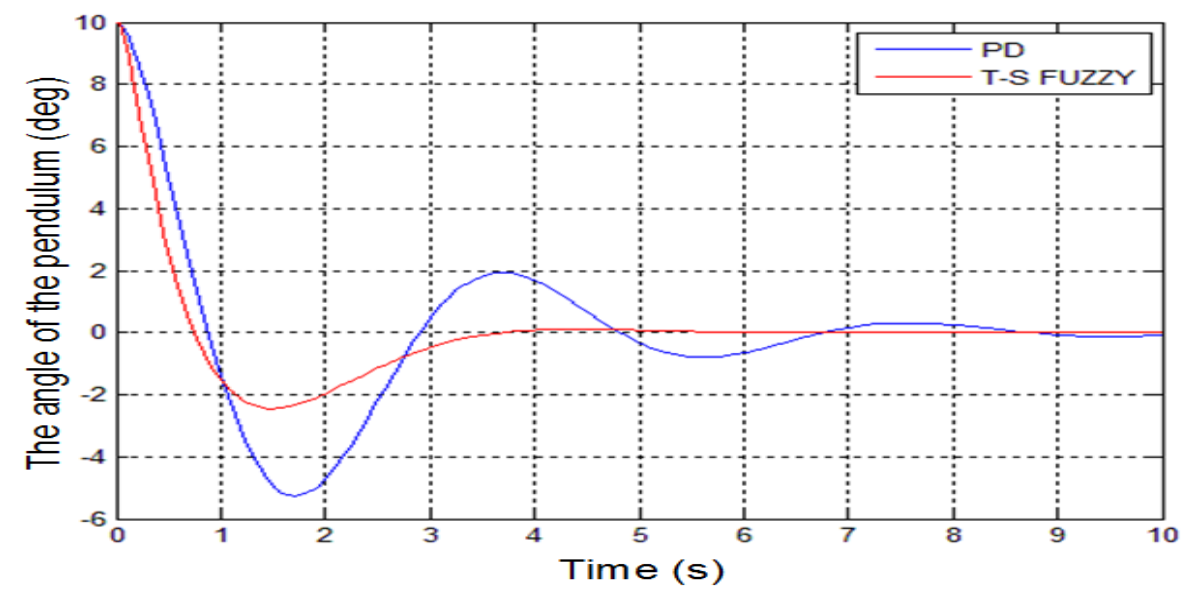

Figure 8. The time response of pendulum angle with PD and T_S fuzzy controller without disturbance.

The time response of pendulum angle with PD and T-S fuzzy controller without disturbance is shown in Fig. 8. It is observed that T-S fuzzy control scheme quickly and smoothly stabilizes the pendulum in a vertically upright position approximately within 4 seconds following a minor undershoot. However, PD control scheme stabilizes the pendulum in a vertically upright position following a major undershoot and a major overshoot. 
Sit at al. / Bitlis Eren Univ J Sci \& Technol / 6(2), p-p, 2016

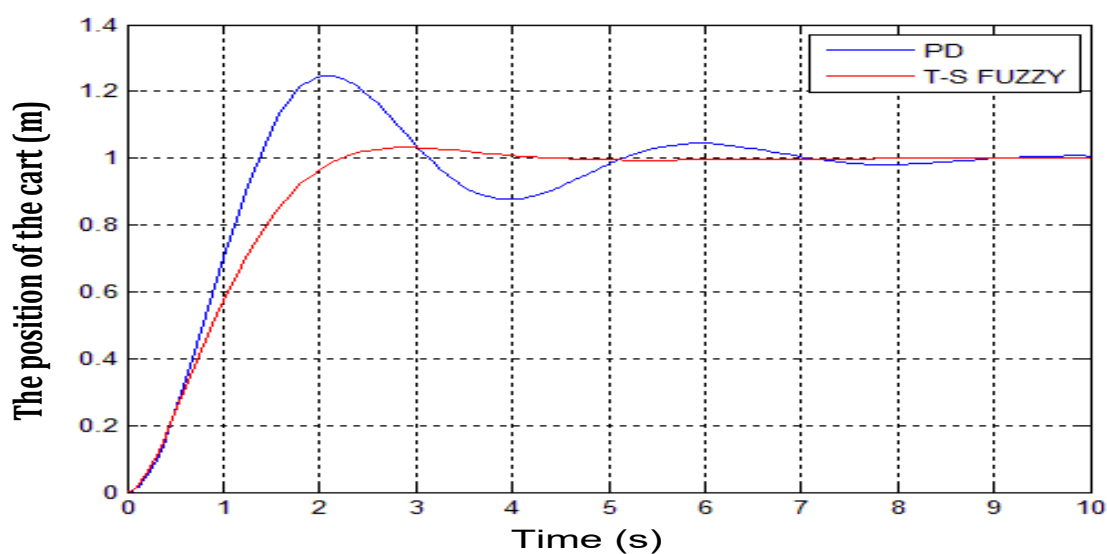

Figure 9. The time response of cart position with PD and Fuzzy controller without disturbance.

The time response of pendulum cart position with PD and T-S fuzzy controller without disturbance is shown in Fig. 9. It is observed that T-S fuzzy control scheme quickly and smoothly stabilizes the pendulum in a vertically upright position approximately within 5 seconds following a minor overshoot. However, PD control scheme stabilizes the pendulum in a vertically upright position following a major undershoot and a major overshoot.

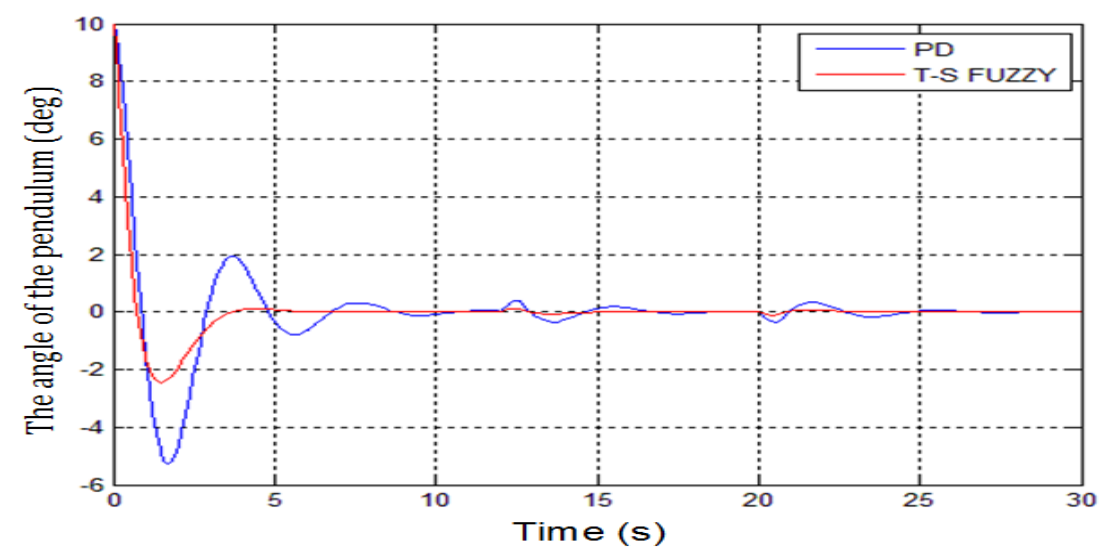

Figure 10. The time response of pendulum angle with PD and Fuzzy controller with disturbance.

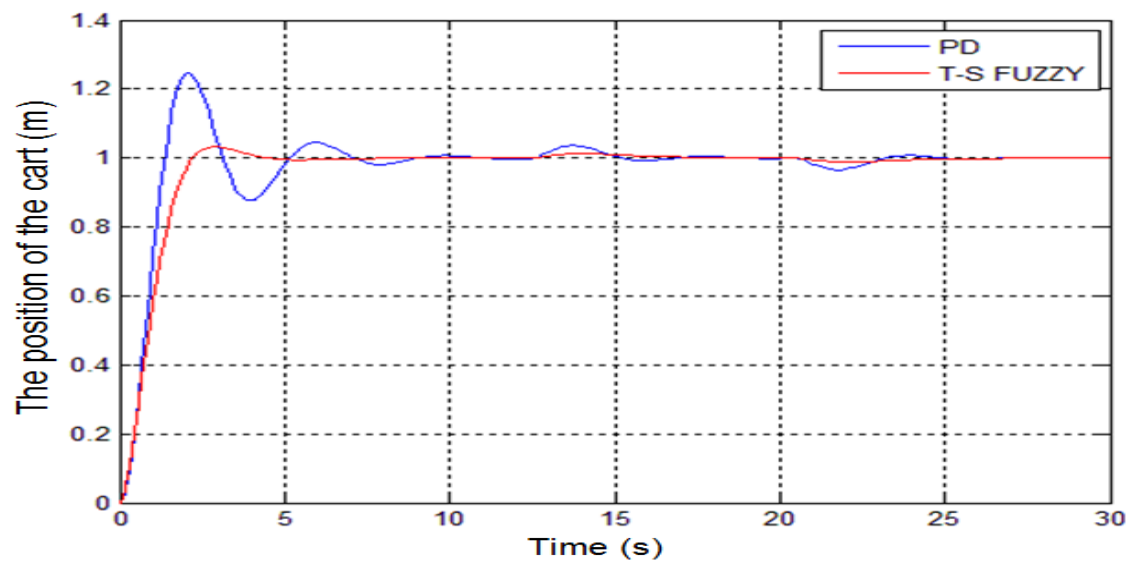

Figure 11. The time response of cart position with PD and Fuzzy controller with disturbance.

The time response of pendulum angle with PD and T-S fuzzy controller with disturbance is shown in Fig. 10. It is observed that T-S fuzzy control scheme quickly and smoothly stabilizes the pendulum in a vertically upright position approximately within 4 seconds following a minor undershoot. PD control scheme stabilizes the pendulum in a vertically upright position following a major undershoot and a major overshoot. The angle of pendulum never oscillates with T-S fuzzy controller before and after disturbance. Before disturbance is applied, PD controller reaches the intended angle minor oscillations approximately within 10 seconds. 
The time response of cart position with PD and T-S fuzzy controller with disturbance is shown in Fig. 11. It is observed that T-S fuzzy control scheme quickly and smoothly stabilizes the pendulum in a vertically upright position approximately within 2 seconds following a minor overshoot. However, PD control scheme stabilizes the pendulum in a vertically upright position following a major undershoot and a major overshoot. The cart position never oscillates with T-S fuzzy controller before and after disturbance. Before disturbance is applied, PD controller reaches the intended position minor oscillations approximately within 10 seconds.

The time response specification for the inverted pendulum system equipped with the two proposed controllers is given in Table 4 and 5, respectively.

Table 4. The time response specification for the angle of the pendulum.

\begin{tabular}{|c|c|c|}
\hline Time response specification & PD & T-S Fuzzy \\
\hline Settling time (s) & 8.6 & 3.8 \\
\hline Rise time (s) & 0.88 & 0.75 \\
\hline Peak overshoot & $2.9 \%$ & $1.3 \%$ \\
\hline Steady state error & 0.02 & 0 \\
\hline
\end{tabular}

Table 5. The time response specification for the position of the cart.

\begin{tabular}{|c|c|c|}
\hline Time response specification & PD & T-S Fuzzy \\
\hline Settling time (s) & 8.6 & 3.8 \\
\hline Rise time (s) & 1.37 & 2.23 \\
\hline Peak overshoot & $24.5 \%$ & $3.3 \%$ \\
\hline Steady state error & 0.01 & 0 \\
\hline
\end{tabular}

\section{Conclusions}

In this study, PD and Takagi-Sugeno type Fuzzy Logic Controller are applied to the inverted pendulum system. Both controllers are compared to determine which one gives a better performance to maintain the pendulum in a balanced state. When the simulation studies are analyzed, the response speed of T-S fuzzy controller is better and its steady state error is zero. On the other hand, settling time of PD controller is longer, and some steady state errors may be observed for this controller before and after disturbance.

A Takagi-Sugeno type Fuzzy Logic Controller may show a better performance by adjusting the membership functions or the rule base using one of the evolutionary algorithms such as a genetic algorithm or particle swarm optimization (Moghaddas, 2012; Juang, 2011). Various control methods such as optimal control, adaptive control, neural networks and sliding mode control can be applied for the inverted pendulum system.

\section{References}

Acikgoz H, Kececioglu OF, Gani A, Sekkeli M (2014). Speed Control of Direct Torque Controlled Induction Motor By using PI, Anti-Windup PI and Fuzzy Logic Controller, International Journal of Intelligent Systems and Applications in Engineering, 2(3), 58-63.
Bhattacharya S, Chatterjee A, Munshi S, (2003). An Improved PID-type Fuzzy Controller Employing Individual Fuzzy P, Fuzzy I and Fuzzy D Controllers, Transactions of the Institute of Measurement and Control 25, 352-372.

Gani A, Acikgoz H, Kilic E, Ozcalik HR (2014a). Dynamic Performance Analysis of Inverted Pendulum System by using PD-Fuzzy Logic Controller and PD Controller, International Refereed Journal of Engineering And Sciences, 1(2), 1-13.

Gani A, Baş Z, Acikgoz H, Kececioglu OF (2014b). Control of Nonlinear Dynamic Inverted Pendulum System Using Fuzzy Logic Based Control Methods, International Journal of Engineering Research \& Technology, 3(9), 1400-1404.

Gani A, Ozcalik HR, Sekkeli M, Kececioglu OF, Sit S (2016). Dynamic Performance Comparison of PD and PD-Fuzzy Logic Controllers for Inverted Pendulum System, International Conference on Natural Science and Engineering (ICNASE'16), Kilis, Turkey.

Goher KM, Tokhi MO (2010). A New Configuration of Two-Wheeled Inverted Pendulum: A LagrangianBased Mathematical Approach, Multidisciplinary Journals in Science and Technology, Journal of Selected Areas in Robotics and Control (JSRC), December Edition. 
Hasan M, Saha C, Rahman MM, Sarker MRI, Aditya SK (2012). Balancing of an Inverted Pendulum Using PD Controller, Dhaka Univ. J. Sci. 60(1), 115-120.

Hellendoom H, Thomas C (1993). Defuzzification in Fuzzy Controllers, Intelligent and Fuzzy Systems, 1, 109-123.

Juang CF and Chang YC (2011). Evolutionary-GroupBased Particle-Swarm-Optimized Fuzzy Controller with Application to Mobile-Robot Navigation in Unknown Environments, in IEEE Transactions on Fuzzy Systems, 19(2), 379-392.

Khosla A, Leena G, Soni MK (2014). Various Control Techniques and their Performance Analysis for Inverted Pendulum, Int. J. on Recent Trends in Engineering and Technology, 11 (1).

Kilic E, Ozcalik HR, Yilmaz S, Sit S (2015). A Comparative Analysis of FLC and ANFIS Controller for Vector Controlled Induction Motor Drive, 2015 IEEE International Aegean Conference on Electrical Machines \& Power Electronics (ACEMP2015), 2-4 September 2015, Side-Antalya, Turkey.

Lee CC (1990). Fuzzy Logic in Control Systems: Fuzzy Logic Controller-Part I, IEEE Transactions on System Man and Cybernetics, SMC-20(2), 404-418.

Marzi H (2005). Multi-Input Fuzzy Control of an Inverted Pendulum using Anarmature Controlled DC Motor, Robotica (2005), Cambridge University Press 23, 785-788.

Moghaddas M, Dastranj MR, Changizi N and Khoori N, (2012). Design of Optimal PID Controller for Inverted Pendulum Using Genetic Algorithm, International Journal of Innovation, Management and Technology, 3(4), August 2012.

Ozcalik HR, Gani A, Kilic E, Kececioglu OF (2014). DC Servo Motor Speed Control in Nonlinear Load by Using Different Fuzzy Membership Functions, Academic Platform Journal of Engineering and Science, 2(3), 1-8.

Sevil, M., Elalmıș, N., Görgün, H., Aydın, N., (2015). Control of Air Conditioning with Fuzzy Logic Controller Design for Smart Home Systems, Sigma Journal of Engineering and Natural Sciences, 33(3), pp. 439-463.

Sugeno, M., (1985). Industrial Applications of Fuzzy Control, Elsevier Science Inc.

Takagi, T., Sugeno, M., (1985). Fuzzy Identification of Systems and Its Applications to Modeling and Control, IEEE Trans. Syst. Man Cybern., 15, pp.116-132.

Wang, S-Y., Tseng, C-L., Chiu, C-J., (2015). Design of a Novel Adaptive TSK-Fuzzy Speed Controller for use in Direct Torque Control Induction Motor Drives, Applied Soft Computing, Vol. 31, 396-404 pp.
Yamakawa $\mathrm{T}$ (1989). Stabilization of an Inverted Pendulum by a High Speed Fuzzy Logic Controller Hardware System, Fuzzy Sets Systems, 32, 161180.

Yaras B, Hüseynov R, Namazov M, Celikkale IE, Seker M (2014). Fuzzy Control and Sliding Mode Fuzzy Control of DC Motor, Sigma Journal of Engineering and Natural Sciences 32, 97-108. 